\title{
Accretion Disks in the Sub-Stellar Realm: Properties and Evolution
}

\section{Ray Jayawardhana}

\author{
University of Toronto
}

\begin{abstract}
It is now well established that young brown dwarfs harbor accretion disks -and thus undergo a $\mathrm{T}$ Tauri phase- similar to their low-mass stellar counterparts. The supporting evidence includes infrared and millimeter observations of the dust component as well as optical and infrared spectra with signatures of gas accretion and outflow. Recent findings suggest that disks are common even around young planetary mass objects. The ubiquity of circum-sub-stellar disks not only hints at a common formation scenario for PMOs, brown dwarfs and stars, but also offers a new regime for investigating processes such as episodic accretion, grain growth and disk clearing.
\end{abstract}

